\title{
Modelling Uptake Sensitivities of Connected and Automated Vehicle Technologies
}

\author{
Gillian Harrison, University of Leeds, UK \\ Simon P. Shepherd, University of Leeds, UK \\ Haibo Chen, University of Leeds, UK
}

\begin{abstract}
Connected and automated vehicle (CAV) technologies and services are rapidly developing and have the potential to revolutionise the transport systems. However, like many innovations, the uptake pathways are uncertain. The focus of this article is on improving understanding of factors that may affect the uptake of highly and fully automated vehicles, with a particular interest in the role of the internet of things (IoT). Using system dynamic modelling, sensitivity testing towards vehicle attributes (e.g., comfort, safety, familiarity) is carried out and scenarios were developed to explore how CAV uptake can vary under different conditions based around the quality of IoT provision. Utility and poor IoT are found to have the biggest influence. Attention is then given to CAV 'services' that are characterized by the attributes explored earlier in the paper, and it is found that they could contribute to a $20 \%$ increase in market share.
\end{abstract}

\section{KEYWORDS}

Connected and Automated Vehicles, Internet of Things, System Dynamics Modelling, Uptake Sensitivities

\section{INTRODUCTION}

Although Connected and Automated Driving (CAD) technologies are currently being developed and integrated into conventional vehicles, the future pathway to highly or fully automated (SAE Levels 4 and 5 (ANSI, 2018)) and connected vehicles, through complex transition periods, is very uncertain. In this study we develop a system dynamics model of connected and automated vehicle (CAV) uptake, adopting a base model of the transition towards car-sharing and highly automated vehicles (AV) in the Netherlands (Nieuwenhuijsen, Correia, Milkis, van Arem, \& van Daalen, 2018). We adapt and extend this model to understand the complexities of the attributes characterizing CAVs through exploring the sensitivities to uptake. Scenarios based on expert opinion and sensitivity testing allow us to examine our research objectives:

- What are the sensitivities of uptake to utility for Level 4-5 CAVs?

- How could IoT based technology services accelerate, enable or enhance CAV uptake?

In this work there is a particular focus on the quality of the "Internet of Things" (IoT), which advanced connected technologies rely upon (characterized by the comfort and safety of the vehicles) 
as well as the utility these vehicles may provide the user (through comfort, safety, familiarity and attractiveness). We further extend the base model to include selected additional connected technology services (Table 1) that could be offered on a commercial basis in CAVs, by applying learning from public trials and surveys of these services.

\section{Table 1. Connected technology services}

\begin{tabular}{|l|l|}
\hline & $\begin{array}{l}\text { A service which alerts the vehicle of upcoming hazards (e.g. potholes, obstructions) on } \\
\text { the motorway (detected by other vehicles) so the vehicle can take corrective actions. }\end{array}$ \\
\hline PIGHWAY PILOT & $\begin{array}{l}\text { A service which matches users wishing to platoon (drive in conveys with small headway } \\
\text { and driven by lead vehicle) on long journeys. }\end{array}$ \\
\hline URBAN DRIVING & $\begin{array}{l}\text { A service which connects to devices in an urban environment (e.g. traffic systems, } \\
\text { pedestrian smartphones) so that the vehicle can take appropriate actions (e.g. yield to } \\
\text { pedestrians). }\end{array}$ \\
\hline
\end{tabular}

We find that the perceived utility of the CAV or services to the users, and poor quality IoT provision may have the largest impact on CAV uptake pathways. Our conclusions can support the development of business exploitation plans and provide guidance for both local and national policy makers.

\section{BACKGROUND}

System Dynamics (SD) modelling has been widely applied in transportation studies (Shepherd, 2014), including topics such as freight (Kumar \& Anbanandam, 2019), inter-city connectivity (Pierce, Shepherd, \& Johnson, 2019) and road traffic safety (Kizito \& Semwanga, 2020). Of specific relevance to this study, there has been much interest in the automobile industry and uptake of new vehicle technologies. Many of these studies have focused on alternative fuels and powertrain transitions. For example, Struben and Sterman (2008) modelled Alternative Fuel Vehicle uptake in California using a stock and flow system dynamics model, building on the Bass diffusion model Bass (1969) with a social exposure and incorporating an established discrete choice model (Brownstone, Bunch, \& Train, 2000). This basic model has been incorporated and extended by numerous authors (Gómez Vilchez \& Jochem, 2019), who not only look at uptake but also environmental and societal impacts (often focused around energy/emissions). Other SD models that were developed to understand the uptake of advanced vehicle technologies include in-car navigation (Kim, 2007), car-sharing services (Geum, Lee \& Park, 2014) and IoT impact on intelligent transportation (Marshall, 2015).

There have been to date a limited number of studies that have used SD to study the uptake or impact of automated vehicles (AV). Stanford (2015) studied the potential impact on total vehicle distance travelled through a successful introduction of automated vehicles, examining key variables and relationships within a broad system. He concluded that unstable responses leading to either automobile or public transit dominance are more likely than a steady, moderate transition towards an even modal split. This work was limited to a series of qualitative CLDs to explore the authors assumptions. The same author was involved in a study similarly focused on the impact on vehicle use and modal shift (Gruel \& Stanford, 2015), and again exploring CLDs of transport systems. Their findings were positive across all their scenarios (safer, better use of time, increased accessibility, 
lower monetary costs and less energy use). A key insight was that AVs alone are unlikely to lead to a sustainable transport system, so early policy intervention to avoid AV dominance is necessary. Nieuwenhuijsen et al. (2018) developed a full SD model to represent the diffusion of all AV levels, driven by technology development and also incorporating the uptake in car-sharing. Through three scenarios (Base, Conservative Bloom and Progressive Bloom) applied to the Netherlands, they concluded that there were high levels of uncertainty, though higher levels of automation are unlikely to gain large market shares (even long-term) without optimistic conditions (mainly related to economics and user perceptions). Using the results from Niewenhuijsen and an established SD-related model 'ScenarioExplorer' (Malone, Verroen, Korver, \& Heyma, 2001), Puylaert, Snelder, van Nes, and van Arem (2018) considered the impacts and uncertainties of low levels of automation (L1-3). They found that traffic and congestion would likely increase, though when cars are also connected there is less congestion despite a higher number of trips. In the final study identified in our research, the established 'MARs' Model (Pfaffenbichler, Emberger, \& Shepherd, 2008) was extended to understand potential effects of AVs on land use and the transport system (May, Shepherd, Pfaffenbichler \& Emberger, 2019). In line with findings in other studies, the authors conclude that under all scenarios tested, total vehicle distance travelled would be expected to rise as AVs were introduced (with contribution from 'empty' trips), and this could lead to reduced public and active transport use, and suggest this could be mitigated by encouraging the adoption of shared vehicles. It is interesting that although these examples are few, there are similar conclusions drawn regarding both the uncertainty and the risk of increased vehicle use as AVs are introduced and become widespread, which is in conflict with aspirations of sustainable transport systems.

\section{MODEL DESCRIPTION}

In this study, we build on the model developed by Nieuwenhuijsen et al. (2018). In this base model, private car ownership is gradually replaced by car-sharing, technological development is driven by research funding and learning, and adoption of technologies is based on vehicle attributes of price, comfort, familiarity and safety. All 6 levels of automation ( $\mathrm{L} 0=$ conventional unautomated to L5 = fully automated) are included in the model, though there is no explicit inclusion of IoT connection. A high-level overview of the key variables and feedbacks is shown in Figure 1, and full model details, including key equations and inputs are available in Nieuwenhuijsen et al. (2018) ${ }^{1}$.

The base model was developed to operate under three scenarios: a Base scenario designed to reflect a "business as usual" situation (ie no major support for CAV) with a base year of 2000, and two optimistic "In Bloom" scenarios (one conservative and one progressive) starting from the year 2015, where conditions are favourable towards AV. In this study, the "In Bloom" Conservative Scenario will be used as the base scenario. The Base scenario is not suitable as L4-5 do not gain significant market share even by 2100 , due to lack of technology maturity and the Progressive scenario was designed to be unrealistically ambitious. By adopting the In Bloom Conservative we are implicitly assuming favourable conditions (economic and political) are in place. One small amendment is made to the base model in that the growth in L3+ models are restricted until a realistic year. In order for the model to run a small number of fleet are needed from the start. However, this led to small yet unrealistic market shares in early years as they were included in the choice set and thus grew in size. Therefore, the L3 vehicles were restricted from the choice set until 2020, L4 until 2022 and L5 2025. Another change is that the model is only run for 50 years until 2050, rather than 2100 as with the original model (longer timeframes induce greater uncertainties).

The installed base fleet shares of our base run are shown in Figure 2. Note that L0 vehicles remain dominant in the early years and do not become insignificant until the 2040s. There is competition between lower levels of automation, with L1, 2 and 3 peaking at years 2026, 2032 and 2042 resp., and L4 and 5 becoming more dominant only after 2045 (accounting for around half of the fleet), with L4 equalling L3 by 2050 (which is decreasing). 
Figure 1. High level overview of the base model (adapted from Figure 2 of Nieuwenhuijsen et al. (2018))

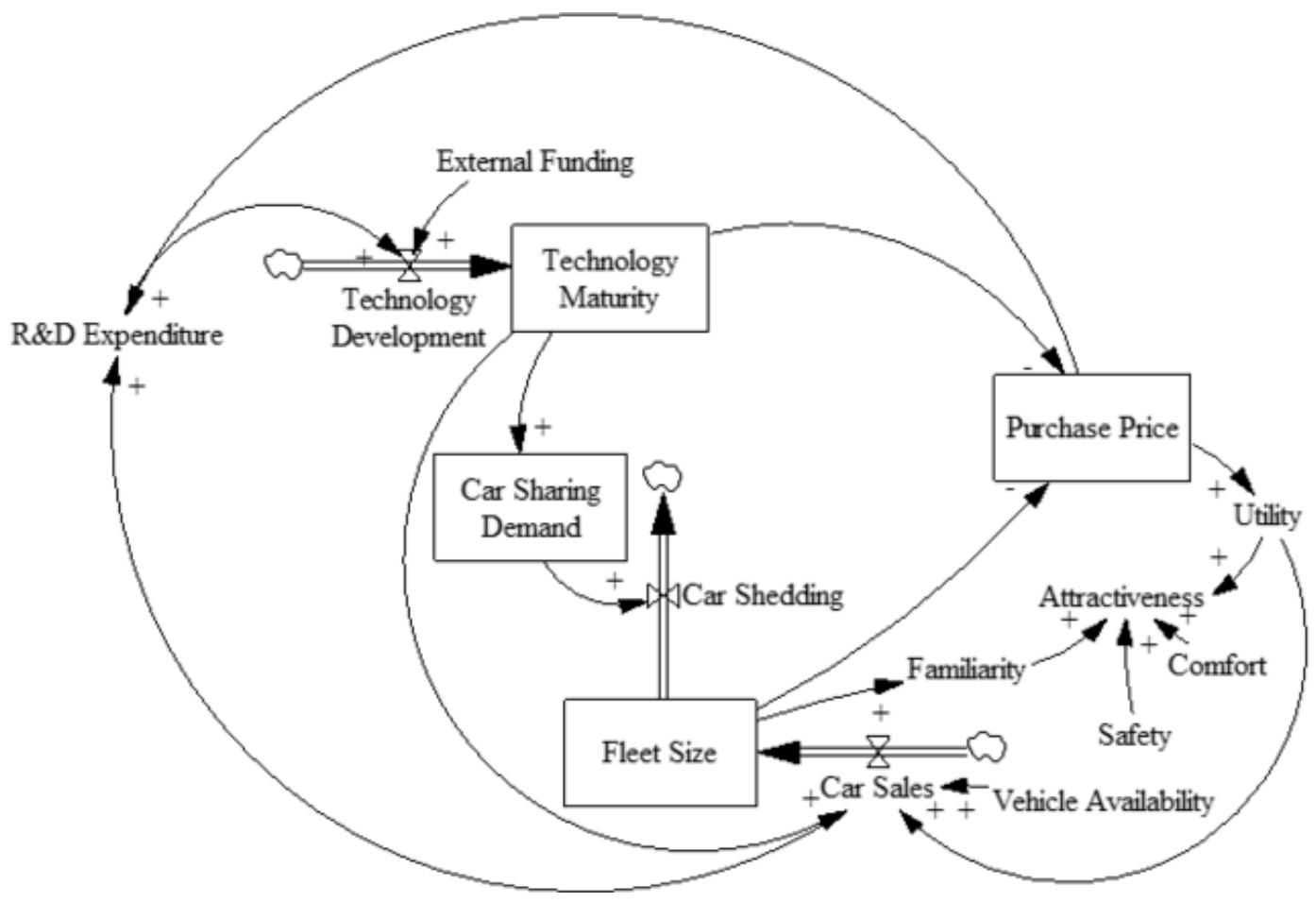

\section{SENSITIVITY TESTING}

The role of IoT and the introduction/uptake of connected technology services is expected to enhance, accelerate and enable the uptake of automated vehicles. Before these are built into the model, it is important to understand which existing variables may offer a connection into a feedback loop that could represent these effects.

Figure 2. Fleet shares of levels of automation to 2050 under our Base conditions (Nieuwenhuijsen et al. (2018) In Bloom Conservative)

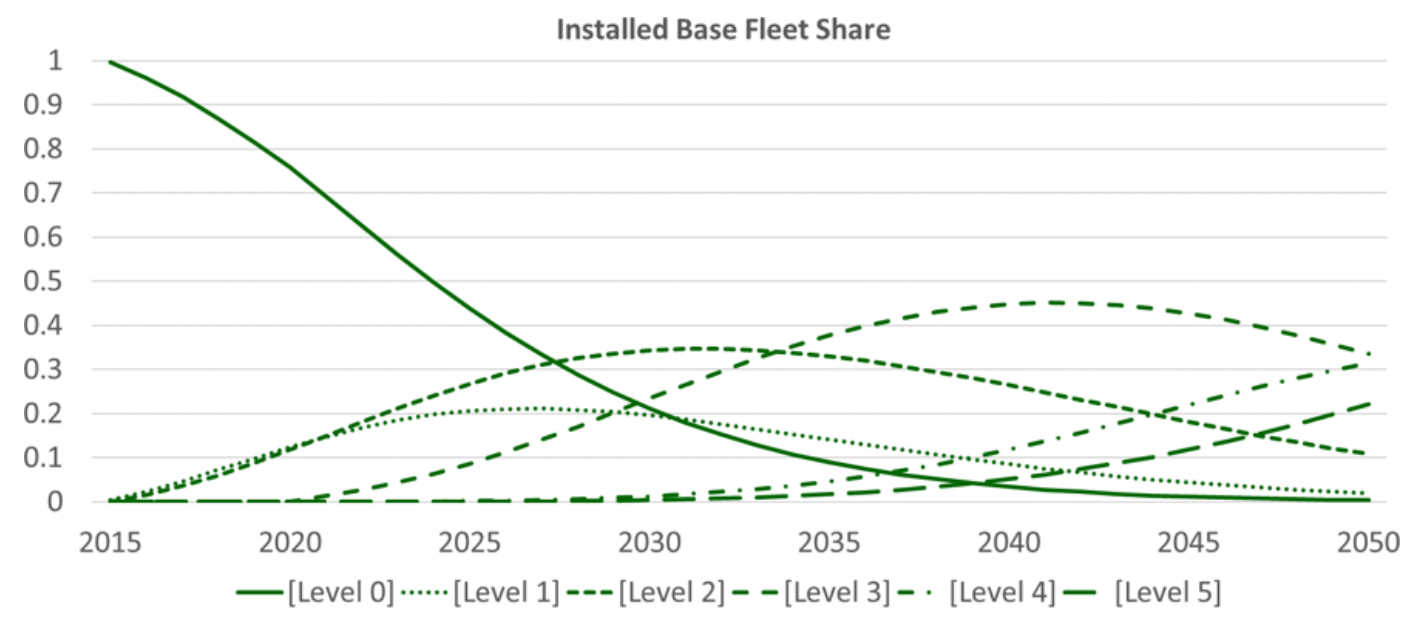




\section{Multivariate}

The sensitivity of fleet shares to variables related to utility and car-sharing was tested, as these could potentially be impacted by the connected technology services. The selected variables, and the sensitivity ranges tested, are described below ${ }^{2}$. The ranges are designed to be extreme without being unrealistic, based on our own assumptions.

\section{Variable Weightings}

Utility $_{\mathrm{L}}=\left(1-\mathrm{P}_{\mathrm{L}}\right) \times \mathrm{W}_{\mathrm{P}}+\left(\mathrm{S}_{\mathrm{L}} \times \mathrm{W}_{\mathrm{S}}+\mathrm{C}_{\mathrm{L}} \times \mathrm{W}_{\mathrm{C}}+\mathrm{F}_{\mathrm{L}} \times \mathrm{W}_{\mathrm{F}}\right) \times \mathrm{W}_{\mathrm{A}}$

$\mathrm{L}=$ Automation Level, $\mathrm{W}=$ Weighting, $\mathrm{P}=$ Normalised Price, $\mathrm{S}=$ Safety, $\mathrm{C}=$ Comfort, $\mathrm{F}=$ Familiarity, $\mathrm{A}=$ Attractiveness

The transition of a user from using a vehicle of one level of automation to another level is driven (in part) by the relative utility of the two levels of automation, using Equation 1. The values of the attributes which determine the utility vary by automation level. The utility comprises of the normalised Price and "Attractiveness" of the vehicle, which are weighted against each other (weights adding up to make "1") according to an importance assigned by the original model authors. The base value of the Attractiveness Weighting is 0.6 (i.e. Price Weighting is therefore 0.4 ). For our sensitivity testing, we have chosen a minimum weighting of 0.5 and a maximum of 0.8 , as price is well known to be highly important so it would not be realistic to go outside these bounds (and were also the 'base' and 'progressive' inputs for the original model scenarios). The value of Attractiveness comprises of "Familiarity", "Safety" and "Comfort", which are also weighted against each other. Taking an assumption that Familiarity (represented by fleet share) also represents confidence and acceptance in new technologies, we implement a weighting sensitivity range of 0.1 to 0.5 for the sensitivity tests, against a base of 0.2. The base value for Safety Weighting is also low at 0.2 , leaving a base Comfort Weight of 0.6. Within the model, we vary these weightings by a ratio between the two, which is 0.25 under base conditions. To assess the sensitivity of fleet share to the weighting given to Safety, the ratio of Safety Weighting to Comfort Weighting is varied between 0.2 and 0.8 .

\section{Safety and Comfort}

The value levels of the attributes of Safety and Comfort are considered to be decimal (varying between minimum 0 and maximum 1) in the model and are specific to each level of automation. They are constant throughout the simulation. The Comfort levels represent the usefulness of time spent in a vehicle, though it can be assumed that it could also represent a level of acceptance and be related to the quality of IoT platforms and technologies. The base values (L3 $=0.5, \mathrm{~L} 4=0.8, \mathrm{~L} 5=1)$ is an average of values given by 15 AV experts (Nieuwenhuijsen, 2015; Nieuwenhuijsen et al., 2018). The maximum and minimum values selected for the sensitivity test are the maximum and minimum values from across the experts. Safety can also be considered to be affected by IoT and related services. The base values for safety were assumed by the original model authors. Maximum and minimum values for our sensitivity tests were chosen in a way that seemed reasonable in terms of the base values.

\section{Adoption Rates of Car Sharing}

Within the base model, car sharing is assumed to grow at an annual rate of $20 \%$, based on a literature review carried out by the original model authors (Nieuwenhuijsen et al., 2018), though they note that this may be high as current users of car-sharing services may be early adopters. In addition to this, an extra growth rate, also of $20 \%$ is assumed once L5 technology is over a maturity of 0.4 . As IoT and related services may support car sharing, a large sensitivity range of $5 \%$ to $40 \%$ is considered for both growth rates. 


\section{Results}

The variables and their ranges as discussed above are summarised in Table 2. Sensitivity tests are an integrated function within Vensim ${ }^{\mathrm{TM}}$ whereby multiple variables can be selected and tested concurrently. In this experiment, 2000 simulations were carried out using a random-uniform montecarlo approach to selecting different variable values for each individual simulation within the given ranges.

Table 2. Ranges of multivariate sensitivity test variables (see descriptions in text for explanations)

\begin{tabular}{|c|c|c|c|}
\hline Variable & Base & Minimum & Maximum \\
\hline Attractiveness & 0.6 & 0.5 & 0.8 \\
\hline Familiarity & 0.2 & 0.1 & 0.5 \\
\hline Ratio of Safety to Comfort & 0.25 & 0.2 & 0.8 \\
\hline Safety L3 & 0.3 & 0.3 & 0.7 \\
\hline Safety L4 & 0.7 & 0.5 & 0.8 \\
\hline Safety L5 & 1.0 & 0.6 & 1 \\
\hline Comfort L3 & 0.5 & 0 & 0.8 \\
\hline Comfort L4 & 0.8 & 0.1 & 0.9 \\
\hline Comfort L5 & 1.0 & 0.7 & 1 \\
\hline Normal growth rate & 0.2 & 0.05 & 0.4 \\
\hline Additional growth rate due to L5 AV & 0.2 & 0.05 & 0.4 \\
\hline
\end{tabular}

Figure 3 shows the results of the multivariate sensitivity test for Levels 4 and 5, and our base run is shown as a single red line. The colour bands show the range in which the fleet share is sensitive to our variables. $100 \%$ of the simulations fall within the entire coloured area (bounded by grey), $95 \%$ within the area bounded by blue, $75 \%$ within the green bounds and 50\% in the yellow shaded area only. Both L4 and L5 have a wide sensitivity range, with the base simulation towards the higher end. This indicates that although the tested variables can contribute to enhancing or enabling AV uptake, they can also lead to inhibited growth. The base scenario 2050 share of L4 is around $30 \%$, which fits fairly evenly between a sensitivity range of 15 to $35 \%$. There is an approx. $20 \%$ L5 base share by 2050. However, the sensitivity tests predict a range of around 10 to $30 \%$.

\section{Scenarios}

To look into the intricacies of the sensitivities identified in the multivariate tests in more detail, a number of illustrative scenarios were carried out, which are presented and discussed in this section. In developing the scenarios, we have divided the variables into 4 groups: IoT Operability, Utility, Safety Weighting and Car Sharing, which may be base level, low or high within a single scenario. The variable ranges are generally the same as within the multivariate sensitivity test, though some changes are reasoned below.

\section{IoT Quality}

The Comfort and Safety attributes (Table 3) are assumed to be influenced by the availability of connected technology services, which in turn depend on the availability and quality of IoT platforms. In the 'low' case, we assume that the provision and quality (ie reliability, speed etc) of the 'connectedness' 
Figure 3. Sensitivity tests under Base conditions

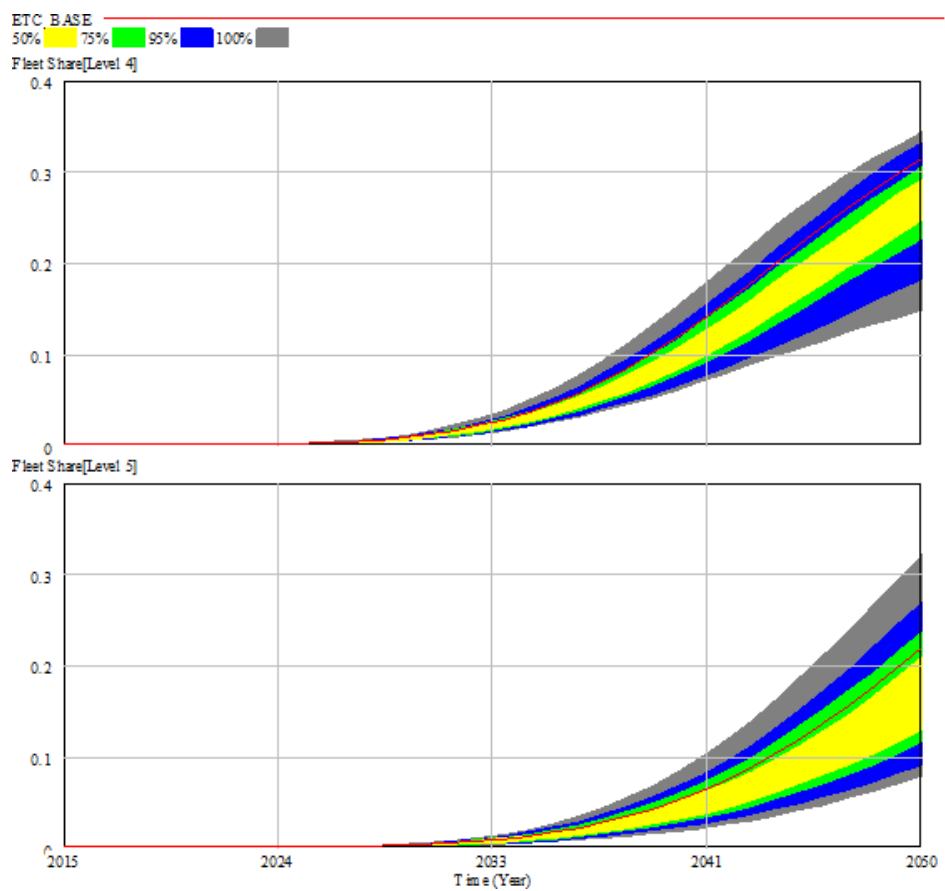

provided by the IoT is poor, whereas in the 'high' case it offers to support more functions than the base value (for L3 and 4). The lower bound for the comfort values have been increased from the multivariate tests, as to be more realistic.

\section{Utility}

The weighting of the utility attributes could represent shifting preferences but also the acceptance of new technologies. Familiarity weighting is at its highest in the 'low' case as this leads to a higher weighting of the Safety and Comfort variables. To maximise the weighting given to Safety and Comfort, it is higher than the multivariate tests, and the attractiveness weighting in the "low" case is lower (Table 4).

Table 3. Comfort and Safety inputs

\begin{tabular}{|c|c|c|c|}
\hline & Base & Poor (IP) & High (IH) \\
\hline Safety L3 & 0.3 & 0.3 & 0.7 \\
\hline Safety L4 & 0.7 & 0.5 & 0.8 \\
\hline Safety L5 & 1 & 0.6 & 0.8 \\
\hline Comfort L3 & 0.5 & 0.3 & 0.9 \\
\hline Comfort L4 & 0.8 & 0.5 & 1 \\
\hline Comfort L5 & 1 & 0.6 & 1 \\
\hline
\end{tabular}


Table 4. Familiarity and Attractiveness Weightings

\begin{tabular}{|c|c|c|c|}
\hline & Base & Low (UL) & High (UH) \\
\hline Familiarity Weighting & 0.2 & 0.7 & 0.1 \\
\hline Attractiveness Weighting & 0.6 & 0.2 & 0.8 \\
\hline
\end{tabular}

\section{Safety to Comfort Ratio}

The weighting of the safety attribute was considered to be at its lowest in the base scenario (0.25), and so only a higher weighting $(0.75)$ was considered within scenarios.

\section{Car Sharing}

It was not felt realistic that car-sharing growth would be higher than $20 \%$, but could be lower (10\%).

\section{Results}

Sixteen scenarios have been developed based on the four input groups described above and are summarized in Table 5. These have been designed to illustrate the potential impacts of each of the input groups. As our main interest in this work is the impact of IoT, these scenarios are based primarily around the IoT input group. The findings are discussed in the next two sub-sections, with the key finding being that (under our modelling and scenario assumptions), utility weighting, which represents technology acceptance, has the greatest impact on automation adoption. Values of Safety and Comfort also have a small impact, dependent on wider conditions.

Table 5. Sensitivity scenarios

\begin{tabular}{|c|c|c|c|c|}
\hline Scenario Name & IoT & Utility & Safety & CS \\
\hline IP & Low & Base & Base & Base \\
\hline IP_CSL & Low & Base & Base & Low \\
\hline IP_UH & Low & High & Base & Base \\
\hline IP_UH_CSL & Low & High & Base & Low \\
\hline IP_UH_SH & Low & High & High & Base \\
\hline IP_UL & Low & Low & Base & Base \\
\hline IP_UL_CSL & Low & Low & Base & Low \\
\hline IP_UL_SH & Low & Low & High & Base \\
\hline IH & High & Base & Base & Base \\
\hline IH_CSL & High & Base & Base & Low \\
\hline IH_UH & High & High & Base & Base \\
\hline IH_UH_CSL & High & High & Base & Low \\
\hline IH_UH_SH & High & High & High & Base \\
\hline IH_UL & High & Low & Base & Base \\
\hline IH_UL_CSL & High & Low & Base & Low \\
\hline IH_UL_SH & High & Low & High & Base \\
\hline
\end{tabular}


Figure 4. Automation fleet share (L4 + L5) under Base conditions and low quality loT

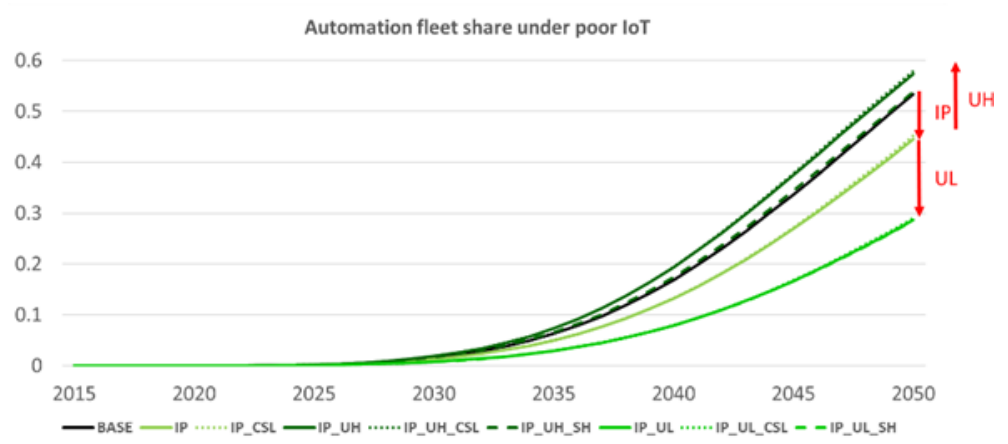

\section{Low Quality IoT}

Under Base conditions, market uptake of automation is already successful so our focus is on what may inhibit or delay uptake. As can be seen in Figure 4, low quality IoT (IP) may cause a small delay, but only noticeably reduce uptake rate when there is also a low value given to vehicle utility (UL). Indeed when Utility is high, there is a marginally greater fleet share in 2050 even under poor IoT. Car sharing and safety weighting have negligible impact. These findings are even more striking when considering L5 only (Figure 5). Here we see that when utility is low the L5 fleet never develops.

\section{High Quality IoT}

As shown in Figure 6, higher quality IoT provision (IH) does not appear to accelerate AV uptake in these conditions. Interestingly, the high quality IoT actually inhibits uptake as it also promotes L3 vehicles, which mature earlier in the simulation. When utility is given low value (UL), shown in Figure 7 uptake can be inhibited even with high quality IoT provision. Again, car sharing and safety weighting have negligible impact. Similar to the poor IoT case, there is market failure for L5 vehicles when utility is valued low.

\section{IMPACT OF CONNECTED TECHNOLOGY SERVICES}

From the sensitivity testing described in the above section, we have determined that the uptake of L4 and L5 CAVs are most sensitive to the attractiveness weighting in the utility and the level of Safety and Comfort. To explore how the connected technology services may accelerate, enhance or enable

\section{Figure 5. L5 fleet share under Base conditions and low quality loT}

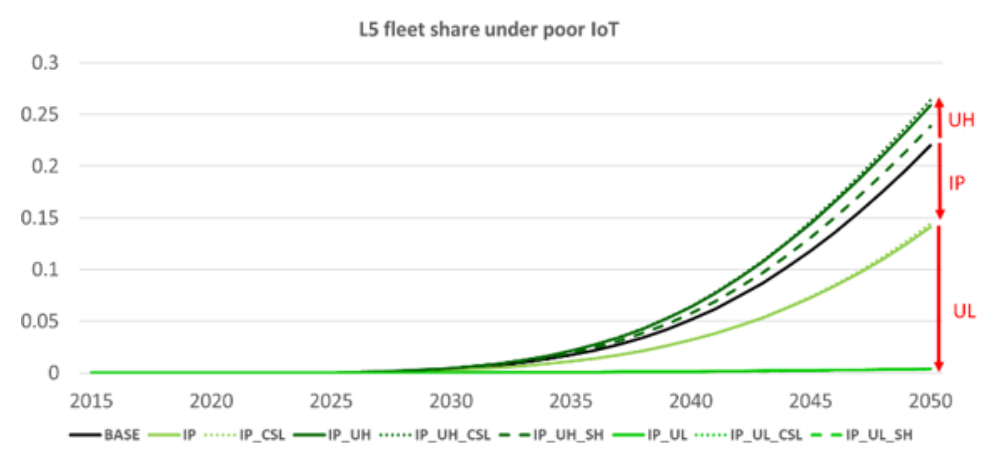


Figure 6. Automation (L4 + L5) fleet share under high quality loT

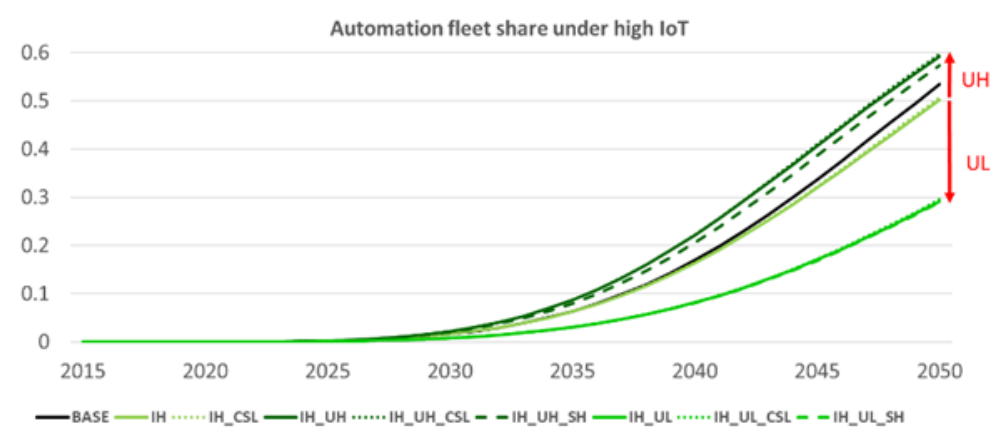

Figure 7. L5 fleet share under and high quality loT

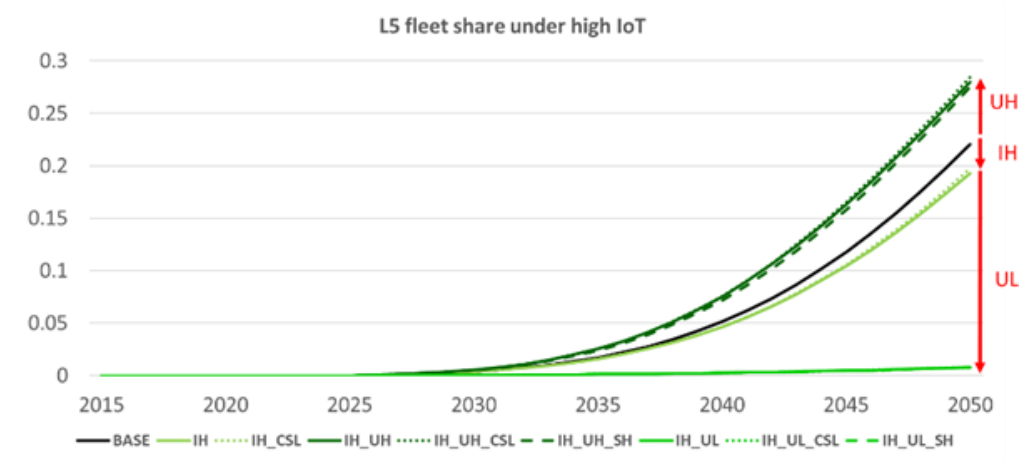

(C)AVs, we extended the model to include the uptake of these. The three services considered in this model, which were shown in Table 1, have been developed within the EU-funded AUTOPILOT (www.autopilot-project.eu) project and been subject to public user testing in Brainport, Netherlands.

The model extension is shown in Figure 8. Fleet Size (by automation level) is calculated within the base model, and the Utility Modifier is the key output of the extension which feeds back into the Utility function within the base model (thus leading to transfer between automation levels as described in the previous section). It is assumed that although all SAE L4/5 vehicles can be equipped with the services, they do not come as standard and a user would choose to use it at extra cost. However, the costs are not directly included in our model, as this data is not available. We recognise that it could reasonably be expected that L5 vehicles should have all three services as standard. However, they were developed within the AUTOPILOT research project as specific value-adding commercial services that users would subscribe to. It is not unusual that a service that is originally introduced as payto-use may become widely available (and relied upon) as a free service once it matures (eg satellite navigation), but this is not directly considered in our model. Scenarios can be run within the model by selecting which services ('use cases') are available as a separate service, with the base scenario having the assumption that all services are included as standard. Thus, with this model extension we are assessing the potential impact of the services that are being developed. 


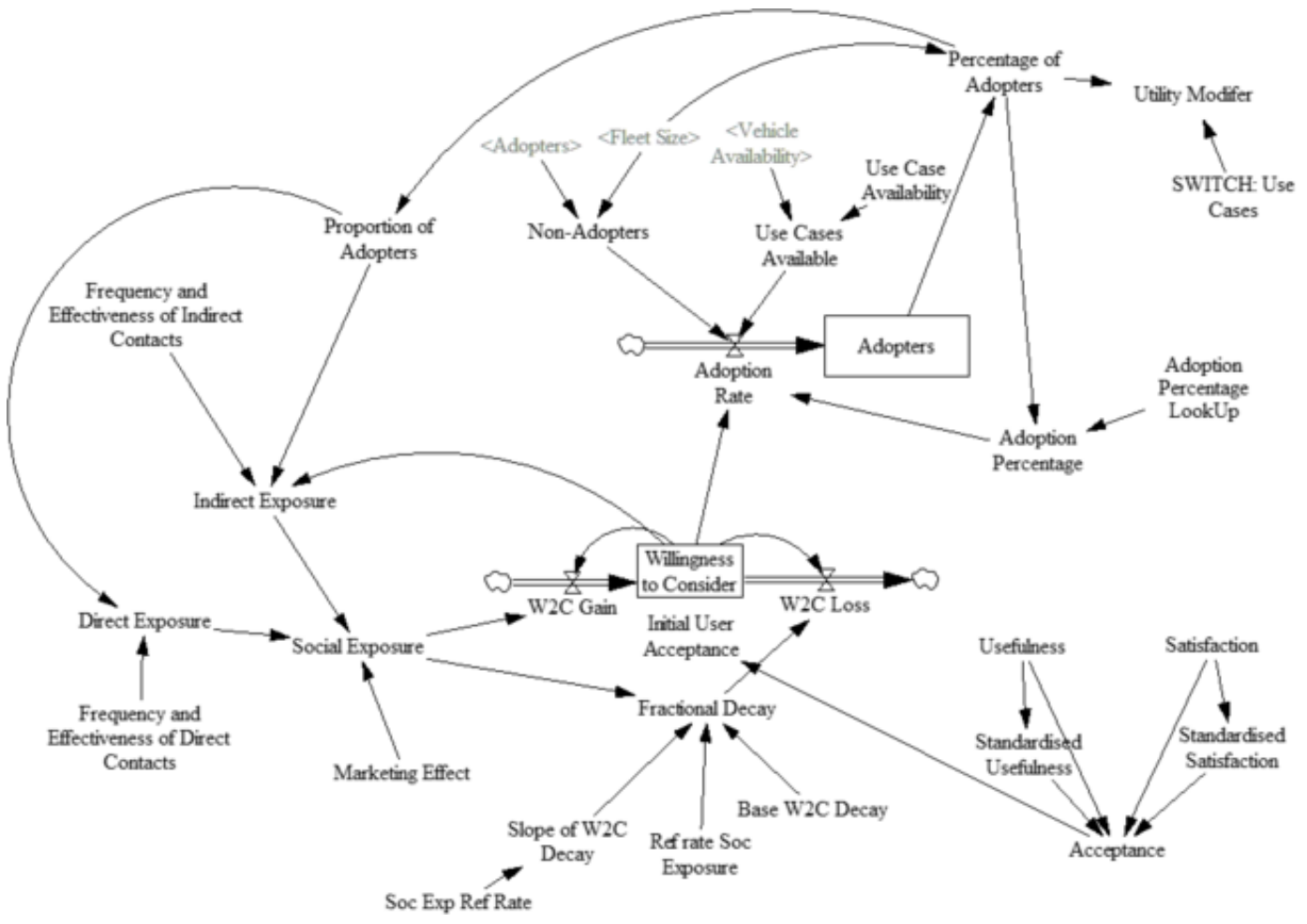

\section{Adoption of Services}

The adoption rate of the services is a product of the number of non-adopters (within the L $4 / 5$ fleet), the 'willingness to consider' the services, and the percentage of those who will use the service once adopted.

Number of non-adopters is based on the fleet size of L4/5 vehicles that are not already adopted. It should be noted that this is vehicles not users, due to structural restrictions of the base model.

Willingness to consider reflects the appearance of the service within a decision set. This can increase over time when influenced by social and media exposure to the service, but can also decay if the exposure is low. The key inputs and structure are based on the willingness to consider electric vehicles (Struben \& Sterman, 2008). The initial user acceptance has been determined using van der Laan scale of technology acceptance (Van der Laan, Heino, \& De Waard, 1997), which determines 'usefulness' and 'satisfaction' from survey data of the three services in public user testing carried out in early 2019 in Brainport, Netherlands. The Methodologies and Analyses of this survey is detailed in Ertl et al. (2019). In summary, participants were asked their opinion (on a 5 point scale) on how they felt about the service after experiencing it in 9 areas that allows an estimation of technology satisfaction and usefulness (useful/useless; pleasant/unpleasant; bad/good; nice/annoying; effective/ superfluous; irritating/likable; assisting/worthless; undesirable/desirable; raising alertness/ sleep inducing). To determine an overall acceptance these results were standardized and an average between satisfaction and usefulness is taken. The values of initial user acceptance determined here are as follows (alongside number of survey participants):

Urban Driving $(\mathrm{n}=43): 0.7$

Highway Pilot $(\mathrm{n}=37): 0.2$ 
Platooning $(\mathrm{n}=20): 0.3$

The percentage of adopters are those who will use the service once they have adopted it. It has also been determined from the public user test surveys (see also Ertl et al. (2019) for full methodologies and results). Users were asked how often they used related existing technology (e.g. navigation systems, cruise control), and this data was used to create a look-up table that related the use of technology (at least once a month) to the population with access to the technology.

\section{Impact of Service Adoption}

The proportion of users of each service is used as a utility modifier. The base model is set up in such a way that the comfort and safety levels are considered to be the maximum value assuming that the vehicle is fully connected and automated. This would only be the case when the full potential of CAVs is met, i.e. when all services are adopted. It is further assumed that the three services contribute equally to the utility. Although it is recognized that there are other services that may contribute, and the three services may not have equal contribution, our interest is focused on the particular services developed within AUTOPILOT. Thus, the attractiveness weight is assumed to have a minimum value of 0.5 and a maximum value of 0.8 , where each service can contribute up to 0.1 . For the comfort and safety levels, there is an assumed minimum equal to the Level 3 values (Safety $=0.3$, Comfort $=0.5$ ), and the maximum is the base value for Levels 4 (Safety $=0.7$, Comfort $=0.8$ ) and Level 5 $($ Safety $=1$, Comfort $=1)$. Each service contributes up to one third of the difference between them.

\section{Results}

Figure 9 shows the uptake of the three AUTOPILOT services by L4 and L5 users (under base conditions). The speed of uptake is affected by the initial user acceptance and the knock on effect that this may have on social exposure. It can be seen that the variance between the service adoption only holds for around 20 years (at around $80 \%$ of adoption), and no services are fully adopted by 2050 .

Figure 10 and Figure 11 show the uptake of automation varies with service availability. We note that the Base scenario gives slightly higher fleet shares then the All Services scenario - this is because the utilities of L4 and L5 vehicles are constant throughout the scenario at the maximum values. In other words, it is a scenario where the services are freely available as standard from the start and are not subject to an uptake curve. The results show that under our Base scenario conditions, the utility provided by all three services could contribute to a market share of highly automated vehicles (L4 and 5) that is 20 percentage points higher by 2050 than a scenario without these services available (No Services), meaning automation would account for over half of the fleet rather than around a third. However, we would not expect the services to be available in this way, but would be commercially

Figure 9. Percentage of L4/5 users who adopt the AUTOPILOT services

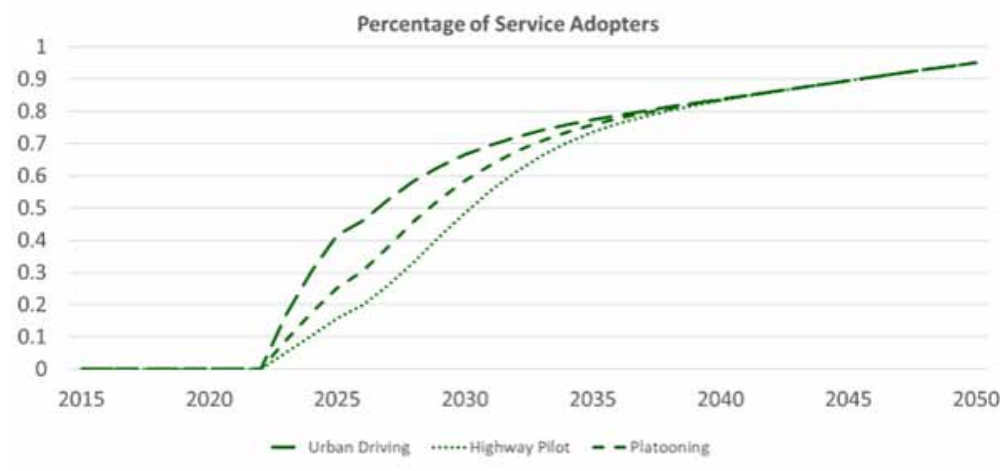


Figure 10. Shares of L4 + 5 vehicles with different AUTOPILOT services available (PT: Platooning; HP: Highway Pilot; UD: Urban Driving)

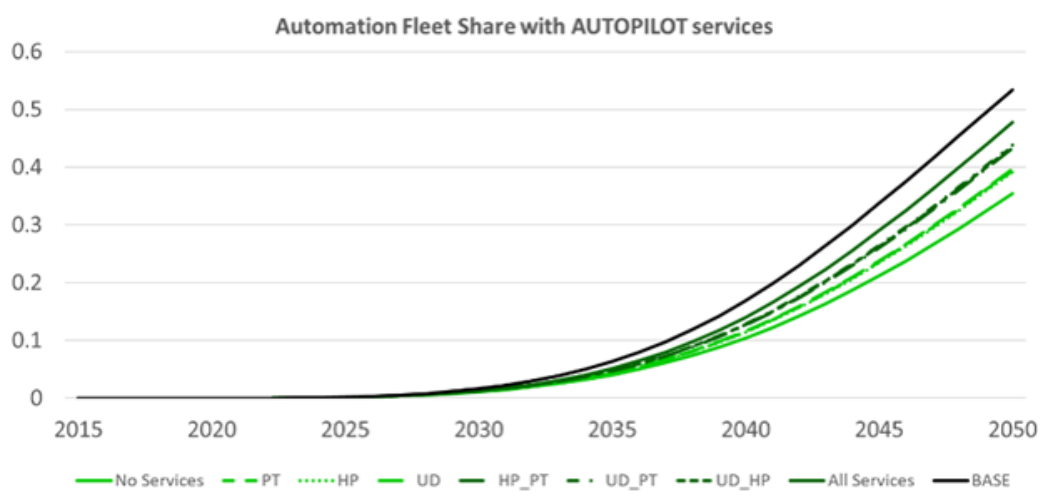

available, thus adoption would increase over time. This All Services scenario would still result in a 2050 AV market share over 10 percentage points higher than No Services. We can also see that (as would be expected), the adoption is dependent on the number of services that are available ( $P T, H P$, $\left.U D, P T \_H P, P T \_U D, H P \_U D\right) T h e s e$ observations are more important for SAE Level 5 vehicles where the absence of services could reduce fleet share to around $5 \%$ by 2050 . We recognise that these services are just illustrative due to the assumptions that we have made, but what we can point to is that services such as these which impact on the utility of AVs are important for sustained market success and will enhance, accelerate and enable the uptake of AVs. For instance, it is arguable that a 5\% share of L5 vehicles 25 years after introduction could at best suggest a niche market or worst be market failure. Thus, for fully automated vehicles to be a market leader (replacing a large fleet proportion) services similar to these, which take advantage of IoT technologies, are necessary.

\section{CONCLUSION}

In this study, we have explored the sensitivity to uptake of the attributes of automated vehicles and extended a base model to include connected technology services. Our findings include:

Figure 11. Shares of L5 vehicles with different AUTOPILOT services availability (PT: Platooning; HP: Highway Pilot; UD: Urban Driving)

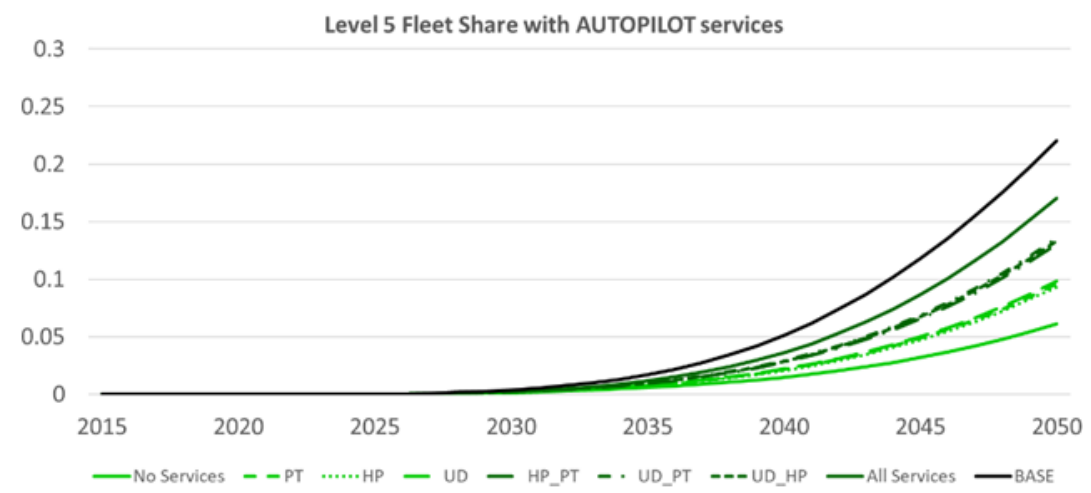


- Poor quality IoT connectivity, platform and services may inhibit market uptake of AV, but this could be overcome by high perceived utility

- $\quad$ IoT connectivity, platform and services mainly impact L3/4 - this could lead to a lowered L5 market share

- Utility has a great effect on uptake and low utility could lead to market failure for L5

- Car sharing and safety value has little impact

What we haven't considered in our work are the wider sustainability implications of the introduction of CAVs, but may draw some qualitative conclusions. This may include both positive and negative impacts on personal mobility, traffic efficiency and wellbeing:

- Personal Mobility may be improved by access to new services and increased vehicle availability but dependent upon business models

- Traffic Efficiency may be improved through increased connected autonomous driving which in turn reduces travel time and improves energy efficiency

- Wellbeing may be improved by less time spent manually driving, reduced emissions and opportunities for new mobility services but may be diminished through impacts on land use, social equity and active transport.

Finally, we can derive the following answers to our research objectives:

- What are the sensitivities to utility of Level 4-5 CAVs?

The results of this study suggest that although IoT connectivity and platforms are important for the uptake of L4-5 CAVs, especially in that it can be inhibited by poor quality IoT, the perceived utility of users has the strongest impact on uptake. Low perceived utility can lead to market failure for fully automated vehicles.

- How could IoT based technology services accelerate, enable or enhance CAV uptake?

It can be deduced that IoT based technology services can accelerate the uptake of CAVs as they contribute to higher levels of perceived utility to the users, both enable and inhibit the uptake of L5 CAVs due to competition with L3 and 4 CAVs and have the potential to enhance certain aspects of CAVs through the provision and integration of additional functionalities, though business models need to be explored to identify the greatest opportunities.

What can be determined from our findings is that of the variables that were selected to represent IoT provision and connected technology services, utility weighting would have the most influence on automated vehicle penetration under all scenarios and conditions. A low utility value can lead to market failure even under Bloom conditions and high quality IoT provision. Although higher quality provision of IoT does not appear to offer great improvements on automated vehicle uptake compared to the base conditions under either Base or Bloom scenarios, it is noticeable that poorer quality IoT could inhibit uptake. This is particularly so for SAE L5 vehicles. Technology developers, service providers and policy makers may all benefit from taking these considerations into account as they develop and introduce CAVs into our transport mix.

\section{ACKNOWLEDGMENT}

The AUTOPILOT project has received funding from the European Union's Horizon 2020 Research and Innovation programme under Grant Agreement No. 731993. The authors would like to thank 
colleague Yvonne Barnard as well as partners on the AUTOPILOT project, in particular the leader of Task 4.4, Elina Aittoniemi of VTT (Finland), who has provided invaluable comments on earlier draft paper, which will form part of the project deliverable. Thanks also go to the authors of the original model on which this study was based, Gonçalo Correia and Jurgen Nieuwenhuijsen, who provided the model and advice in the early stages. 


\section{REFERENCES}

ANSI. (2018). SAE Levels of Driving Automation. ANSI.

Bass, F. (1969). A New Product Growth for Model Consumer Durables. Management Science, 15(5), 215-227. doi:10.1287/mnsc. 15.5 .215

Brownstone, D., Bunch, D. S., \& Train, K. (2000). Joint mixed logit models of stated and revealed preferences for alternative-fuel vehicles. Transportation Research Part B: Methodological, 34(5), 315-338. doi:10.1016/ S0191-2615(99)00031-4

Ertl, D., Harrison, G., Kolarova, V., Barnard, Y., Malin, F., Touliou, K., \& Aittoniemi, E. (2019). AUTOPILOT Deliverable 4.8: User Requirements Analysis. Retrieved from Available from: https://autopilot-project.eu/ deliverables/

Geum, Y., Lee, S., \& Park, Y. (2014). Combining technology roadmap and system dynamics simulation to support scenario-planning: A case of car-sharing service. Computers \& Industrial Engineering, 71, 37-49.

Gómez Vilchez, J. J., \& Jochem, P. (2019). Simulating vehicle fleet composition: A review of system dynamics models. Renewable \& Sustainable Energy Reviews, 115, 109367. doi:10.1016/j.rser.2019.109367

Gruel, W., \& Stanford, J. (2015). Assessing the Long-Term Effects of Autonomous Vehicles: a speculative approach. Paper presented at the European Transport Conference 2015.

Kim, Y. M. (2007). A System Dynamics Model for the Technological Forecasting of Automotive in-car Navigation Market. Paper presented at the 2007 International Forum on Strategic Technology, Ulaanbaatar, Mongolia.

Kizito, A., \& Semwanga, A. R. (2020). Modeling the Complexity of Road Accidents Prevention: A System Dynamics Approach. International Journal of System Dynamics Applications, 9(2), 24-41. doi:10.4018/ IJSDA.2020040102

Kumar, A., \& Anbanandam, R. (2019). Multimodal Freight Transportation Strategic Network Design for Sustainable Supply Chain: An OR Prospective Literature Review. International Journal of System Dynamics Applications, 8(2), 19-35. doi:10.4018/IJSDA.2019040102

Malone, K. M., Verroen, E., Korver, W., \& Heyma, A. (2001). The Scenario Explorer for Passenger Transport: A Strategic Model for Long-term Travel Demand Forecasting. Innovation (Abingdon), 14(4), 331-353. doi:10.1080/13511610120106138

Marshall, P. (2015). System dynamics modeling of the impact of Internet-of-Things on intelligent urban transportation. Paper presented at the 2015 Regional Conference of the International Telecommunications Society (ITS), Los Angeles, CA.

May, A. D., Shepherd, S., Pfaffenbichler, P., \& Emberger, G. (2019). The potential impacts of automated cars on urban transport: an exploratory analysis. Paper presented at the World Conference on Transport Research - WCTR 2019, Mumbai.

Nieuwenhuijsen, J. (2015). Diffusion of Automated Vehicles. A quantitative method to model the diffusion of automated vehicles with system dynamics $(M S c)$. Delft University of Technology.

Nieuwenhuijsen, J., Correia, G. H. A., Milkis, D., van Arem, B., \& van Daalen, E. (2018). Towards a quantitative method to analyse the long-Term innovation diffusion of automated vehicles technology using system dynamics. Transportation Research Part C, Emerging Technologies, 86, 300-327. doi:10.1016/j.trc.2017.11.016

Pfaffenbichler, P., Emberger, G., \& Shepherd, S. (2008). The Integrated Dynamic Land Use and Transport Model MARS. Networks and Spatial Economics, 8(2), 183-200. doi:10.1007/s11067-007-9050-7

Pierce, D., Shepherd, S., \& Johnson, D. (2019). Modelling the Impacts of Inter-City Connectivity on City Specialisation. International Journal of System Dynamics Applications, 8(4), 47-70. doi:10.4018/ IJSDA.2019100104

Puylaert, S., Snelder, M., van Nes, R., \& van Arem, B. (2018). Mobility impacts of early forms of automated driving - A system dynamic approach. Transport Policy, 72, 171-179. doi:10.1016/j.tranpol.2018.02.013 
Shepherd, S. P. (2014). A review of system dynamics models applied in transportation. Transportmetrica B. Transport Dynamics, 2(2), 83-105. doi:10.1080/21680566.2014.916236

Stanford, J. (2015). Possible Futures for Fully Automated Vehicles: Using Scenario Planning and System Dynamics to Grapple with Uncertainty (PhD Thesis).

Struben, J., \& Sterman, J. D. (2008). Transition challenges for alternative fuel vehicle and transportation systems. Environment and Planning. B, Planning \& Design, 35(6), 1070-1097. doi:10.1068/b33022t

Van der Laan, J. D., Heino, A., \& De Waard, D. (1997). A simple procedure for the assessment of acceptance of advanced transport telematics. Transportation Research Part C, Emerging Technologies, 5(1), 1-10. doi:10.1016/ S0968-090X(96)00025-3

\section{ENDNOTES}

The model was developed in Vensim ${ }^{\mathrm{TM}}$ (www.vensim.com) and run with a time step of 0.015625 years. Note all 'Base' values were determined by the original model authors. 


\section{APPENDIX: EQUATIONS OF MODEL EXTENSION}

Acceptance[Use Case] $=($ Standardised Satisfaction[Use Case]*(Satisfaction[Use Case]/2) + Standardised Usefulness〉[Use Case]*(Usefulness[Use Case]/2))/2

Adopters[Use Case]= INTEG (Adoption Rate[Use Case],0)

Adoption Percentage[Use Case]=Adoption Percentage LookUp(Percentage of Adopters[Use Case]) Adoption Percentage LookUp $([(0,0)-10,10)],(0,0.6),(0.2,0.65),(0.4,0.75),(0.6,0.8),(0.8,0.9),(1,1))$

Adoption Rate[Use Case]=("Non-Adopters"[Use Case]*Use Cases Available[Use Case]*Willingness to Consider[Use Case]*Adoption Percentage[Use Case])

Base W2C Decay $=0.15$

Direct Exposure[Use Case]=Proportion of Adopters[Use Case]*Frequency and Effectiveness of Direct Contacts[Use Case]

Fractional Decay[Use Case]=Base W2C Decay* ZIDZ(exp(-4*Slope of W2C Decay*(Social Exposure[Use Case]- Ref rate Soc Exposure)), (1+exp(-4*Slope of W2C Decay*(Social Exposure[Use Case]-Ref rate Soc Exposure))))

Frequency and Effectiveness of Direct Contacts[Use Case] $=0.25$

Frequency and Effectiveness of Indirect Contacts[Use Case] $=0.15$

Indirect Exposure[Use Case]=Frequency and Effectiveness of Indirect Contacts[Use Case]*Proportion of Adopters[Use Case]*Willingness to Consider[Use Case]

Initial User Acceptance[Use Case]=Acceptance[Use Case]

Marketing Effect[Use Case] $=0.025$

“Non-Adopters"[Use Case]=MAX(0,(SUM(Fleet Size["L4-5”!])-Adopters[Use Case]))

Percentage of Adopters[Use Case]=IF THEN ELSE((Adopters[Use Case]/(SUM(Fleet Size[“L4-5”!])))>1, 1, (Adopters[Use Case]/(SUM(Fleet Size[“L4-5”!]))))

Proportion of Adopters[Use Case]=Percentage of Adopters[Use Case]

Ref rate Soc Exposure $=0.025$

Satisfaction[Use Case] $=1.41,1.01,1.4$

Slope of W2C Decay=ZIDZ(1,2*Soc Exp Ref Rate)

Soc Exp Ref Rate $=0.05$

Social Exposure[Use Case]=Direct Exposure[Use Case]+Indirect Exposure[Use Case]+Marketing Effect[Use Case]

Standardised Satisfaction[Use Case] $=($ Satisfaction[Use Case] - VMIN(Satisfaction[Use Case!])) / (VMAX(Satisfaction[Use Case!]) - VMIN(Satisfaction[Use Case!]))

Standardised Usefulness[Use Case]=(Usefulness[Use Case] - VMIN(Usefulness[Use Case!])) / (VMAX(Usefulness[Use Case!]) - VMIN(Usefulness[Use Case!]))

"SWITCH: Use Cases"=0

Use Case Availability[Use Case] $=0,0,0$

Use Cases Available[Use Case]=Vehicle Availability[Level 4]*Use Case Availability[Use Case]

Usefulness[Use Case] $=1.26,1.22,1.16$

Utility Modifer[Use Case]=

IF THEN ELSE("SWITCH: Use Cases" = 0, 1, Percentage of Adopters[Use Case])

W2C Gain[Use Case]=Use Cases Available[Use Case]*(Social Exposure[Use Case]*MAX(1Willingness to Consider[Use Case], 0))

W2C Loss[Use Case]=Use Cases Available[Use Case]*(Fractional Decay[Use Case]*Willingness to Consider[Use Case])

Willingness to Consider[Use Case]= INTEG (W2C Gain[Use Case]-W2C Loss[Use Case],Initial User Acceptance[Use Case]) 
Gillian Harrison (PhD) is an experienced transport policy researcher, with a committed professional interest in low carbon vehicle technologies and sustainable mobility. Specialisms include system dynamics modelling, automobility and policy appraisal. Dr Harrison has six years of business experience in environmental management, project and data co-ordination (in the UK water industry), holds a MSc/PhD in low carbon technologies (modelling, policy and ethics of low carbon vehicles) and three years of post-doctoral research for the European Commission, Joint Research Centre in electro-mobility modelling and policy advice. Since April 2017, Dr Harrison has been employed as a Post-Doctoral Research Fellow at the Institute for Transport Studies, University of Leeds, supporting EU Horizon 2020 and UK funded projects on future mobility, including automated \& connected vehicles.

Simon P. Shepherd is currently the Director of the Institute for Transport Studies, which he has been with since 1989, Prof. Shepherd has worked on and led various EC and UK funded projects. His expertise lies in modelling and policy optimisation ranging from detailed simulation models through to assignment to strategic land use transport models. Prof. Shepherd gained his doctorate in 1994 applying state-space methods to the problem of traffic responsive signal control in over-saturated conditions. His work on optimisation continues at all levels and he continues to work on developing the dynamic LUTI model MARS (with partners in Vienna) providing a systems dynamics approach to strategic modelling. He is currently leading on the development of the Institute's system dynamics expertise.

Haibo Chen $(P h D)$ is a Principal Research Fellow in the Institute for Transport Studies at the University of Leeds. His research is concentrated on applying advanced statistical, mathematical and computing techniques to the analysis of traffic and other transport data for a variety of applications such as incident detection, traffic forecasting and the estimation of vehicle fuel consumption and emissions. Dr Chen has also worked on the application of neural networks within the transport field. He has conducted and managed a wide range of projects funded by various funding bodies. 